\title{
Avaliação e Seleção de Fundos de Investimento: um Enfoque sobre Múltiplos Atributos
}

\author{
Paulo Sergio Ceretta \\ Newton C. A. da Costa Jr.
}

\section{RESUMO}

Este trabalho investiga o desempenho de fundos de investimento em ações mediante a análise por envoltório de dados. Essa ferramenta mostra-se poderosa na avaliação do desempenho de fundos de investimento, quando a função utilidade esperada do investidor apresenta múltiplos atributos. Desta maneira, além dos tradicionais atributos risco e retorno calculados sobre um determinado intervalo de tempo, a análise por envoltório de dados permite incorporar outros atributos importantes ao investidor, tais como custos de administração, restrições de capital e mesmo os atributos risco e retorno calculados em períodos mais recentes. Foram analisados 106 fundos na modalidade carteira livre, no período de dezembro de 1997 a novembro de 1999. Os resultados obtidos identificaram sete fundos dominantes, os quais foram confrontados com os sete fundos menos eficientes, evidenciando-se suas diferenças em termos de atributos e ponderações. Também foi realizada uma comparação em relação ao índice de Sharpe. Cabe salientar que o principal objetivo do presente trabalho não é prescrever uma alternativa para o modelo de otimização média-variância, mas o de descrever uma nova aplicação da análise por envoltório de dados.

Palavras-chaves: fundos de investimento; função utilidade; análise por envoltório de dados.

\begin{abstract}
This work investigates mutual fund performance through data envelopment analysis. The data envelopment analysis has shown to be a powerful tool in the evaluation of investment performance when investor's expected utility function contains multiple attributes. Besides the traditional attributes risk and return calculated over a certain time interval, data envelopment analysis can incorporate other attributes to the investor such as expenses, capital constraints, and even the attributes risk and return calculated over different time periods. We analyzed 106 funds in the period of December 1997 to November 1999. The results identified seven dominant funds which were confronted with the less efficient ones in terms of attributes and weighted differences. We made also a comparison with the results using the Sharpe Ratio. It is worthwhile mentioning that the main objective of this paper is not to prescribe an alternative technique for the mean-variance optimization, but to describe a new application of data envelopment analysis.
\end{abstract}

Key words: mutual fund; utility function; data envelopment analysis. 


\section{INTRODUÇÃO}

Tema central em finanças é a necessidade de haver uma relação de troca (trade-off) entre risco e retorno esperado. Assim, investimentos com elevado nível de risco deverão apresentar indícios de uma justa remuneração; caso contrário, não conseguirão atrair recursos de investidores racionais (avessos ao risco). É de se esperar que um investidor avesso ao risco selecione, entre duas alternativas de mesmo retorno esperado, aquela que apresentar o menor nível de risco ou, entre duas alternativas de mesmo nível de risco, aquela que apresentar o maior retorno esperado.

Embora possa parecer um processo simplista, identificar a melhor opção de investimento é um trabalho árduo e que, em muito casos, apresenta resultados não desejados. Os dois principais aspectos que contribuem para a obtenção de resultados indesejáveis na seleção de alternativas de investimento são: (1) restrito conjunto de informações utilizadas na análise e (2) o comportamento do investidor. A grande maioria das medidas utilizadas na avaliação de alternativas de investimento, tais como o índice de Treynor, índice de Sharpe, medida de Jensen, entre outras, consideram um conjunto muito restrito de informações relacionadas a um único período de tempo, como, por exemplo, o nível de risco e a taxa de retorno calculados sobre determinado período de tempo. Essas informações geralmente são confrontadas entre si ou comparadas com um índice representativo do mercado como benchmark. Tal procedimento é amplamente utilizado e em muito contribui para o refinamento e evolução da teoria de finanças.

Contudo, considerando-se apenas informações relativas às taxas de retorno e ao nível de risco das alternativas de investimento e de um índice de mercado, ignora-se por completo a ação direta dos custos sobre o desempenho do investimento. Assim, alternativas com idênticas taxas de retorno e níveis de risco, mas com distintos custos, seriam consideradas como de mesmo desempenho pelo investidor. Outro fato é que os investidores podem dar maior importância ao comportamento mais recente do investimento (por exemplo, o último ano) e menor importância ao comportamento do investimento num período mais longo de tempo (por exemplo, os últimos dois ou três anos). Se a medida de avaliação não possibilita o fracionamento do período em subperíodos, ela acaba por diluir o impacto de eventos mais recentes, os quais, por ainda estarem na memória do investidor, tendem a exercer maior influência sobre a tomada de decisão. 
Atrelado à utilização de um conjunto restrito de informações está o aspecto comportamental do investidor. Não há consenso sobre como os investidores tomam as suas decisões, embora a teoria de finanças declare que o investidor racional busca a diversificação com objetivos de minimizar o risco para determinado nível de retorno esperado. Modelos de minimização de risco ou de maximização de retorno (Markowitz, 1952), razões simples de retorno/risco (Treynor, 1965; Sharpe, 1966), regressões com índices de mercado (Jensen, 1968) e muitos outros modelos, entre os quais se destacam os de Grinblatt e Titman (1993) e de Grahan e Harvey (1994), são aceitáveis em avaliações em que são consideradas exclusivamente informações sobre risco e retorno; mas, assumindo-se que os investidores consideram um conjunto mais amplo de informações e que informações mais recentes podem exercer influência diferenciada sobre a tomada de decisão do investidor, tais modelos têm a sua eficácia sensivelmente reduzida.

Por considerar um conjunto amplo de informações, muitas vezes o investidor agrega ao seu portfolio títulos que não contribuem, necessariamente, para o aumento do retorno esperado e muito menos para a redução do nível de risco. Esse aspecto é discutido no artigo de Fisher e Statman (1997), no qual é feita uma analogia entre otimização média-variância (Markowitz, 1952) e dieta alimentar. Os autores argumentam que os indivíduos procuram equilibrar as suas dietas entre um grande número de alimentos, sujeitos a restrições de custos e nutrientes; porém, em muitos casos, determinados alimentos, como, por exemplo, o chocolate (que é caro e muito calórico), conseguem seduzir o consumidor, levando-o a aumentar a sua participação na alimentação, desequilibrando-a em termos de custos e nutrientes. De forma idêntica, no mercado financeiro, alguns títulos apresentam maior grau de sedução sobre os investidores do que outros, levando-os, inconscientemente, a incorporá-los em seus portfolios sem devida análise da relação risco-retorno-custo ou de suas correlações com outros títulos anteriormente adquiridos.

Assim, parece ficar claro que a escolha do investidor por determinado investimento é uma decisão que envolve múltiplos atributos. Essa decisão pode estar associada a um conjunto amplo de informações, como as seguintes: risco e retorno em período recente; risco e retorno em período maior de tempo; custos envolvidos; investimento mínimo necessário; movimentação mínima etc. Essas informações são todas economicamente justificáveis, o que, segundo Strong (1993), torna a seleção de um investimento uma decisão de caráter individual e muito específica, muito distante de ser uma decisão simplista, baseada em dois atributos como risco e retorno. Esse, também, é o enfoque apresentado no trabalho de Mcmullen e Strong (1998). 
Nesta pesquisa, é utilizada a análise por envoltório de dados para investigar e analisar comparativamente o desempenho de 106 fundos mútuos de investimento carteira livre. As informações utilizadas compreendem o retorno médio e o desvio padrão (medida de risco) em dois períodos distintos, um período recente de um ano e outro mais amplo de dois anos, além de considerar diretamente a influência do custo de administração sobre o desempenho do fundo. É realizada uma comparação entre os fundos eficientes e os menos eficientes, em termos dos seus atributos e suas estruturas específicas de ponderações e, também, em relação ao índice de Sharpe.

A seguir são apresentadas as principais características da técnica denominada análise por envoltório de dados. Na terceira parte do trabalho, são apresentados os dados e aspectos metodológicos; na quarta parte, apresentam-se os resultados e, por último, a conclusão.

\section{AnÁlise por EnVoltório de DAdos}

A análise por envoltório de dados (data envelopment analysis - DEA) é uma técnica da área de pesquisa operacional, desenvolvida por Charnes, Cooper e Rhodes (1978) e por Banker, Charnes e Cooper (1984). É poderosa ferramenta gerencial, utilizada para avaliação e comparação de unidades organizacionais. A DEA auxilia no processo de tomada de decisão por considerar conjuntamente um grande número de informações, as quais são ponderadas e convertidas em um único escore global, freqüentemente referenciado como indicador de eficiência.

Na prática, a DEA identifica a eficiência de um fundo de investimento, comparando-o com o melhor desempenho observado no seu nível de operação. Ao conjunto de melhores desempenhos dá-se o nome de fronteira de melhor prática ou fronteira eficiente. Essa, por sua vez, elimina a necessidade de se utilizar um índice representativo do mercado ou comportamento médio do setor como benchmark.

Como resultado da aplicação da análise por envoltório de dados, tem-se a classificação das unidades avaliadas (empresas, setores, investimentos etc.) em dois grupos: eficientes e ineficientes. Nesse caso, a palavra eficiência tem uma interpretação semelhante à teoria da carteira de Markowitz. Para Markowitz (1952) um portfolio eficiente é aquele que apresenta o máximo de retorno esperado para determinado nível de risco, ou o menor nível de risco para determinado retorno esperado. Esse portfolio é identificado por meio de um problema de programação quadrática que utiliza critérios de retorno médio e variância. 
$\mathrm{Na}$ análise por envoltório de dados, um portfolio eficiente é aquele que apresenta o máximo nível de atributos desejáveis para determinado nível de atributos indesejáveis ou, de forma contrária, o menor nível de atributos indesejáveis para determinado nível de atributos desejáveis. Esse portfolio é identificado por meio de um problema de programação linear, utilizando-se de múltiplos critérios. $\mathrm{O}$ índice ponderado de performance (IPP), resultante da análise por envoltório de dados, é obtido de uma razão entre a soma ponderada de atributos desejáveis e a soma ponderada de atributos indesejáveis, conforme Equação [1].

$$
I P P_{k}=\frac{u_{1} y_{1}+u_{2} y_{2}}{v_{1} x_{1}+v_{2} x_{2}+v_{3} x_{3}+c}
$$

onde $I P P_{k}$ é o índice ponderado de performance do fundo $k ; y_{1}$ e $y_{2}$ são as taxas de retorno mensal médio em um e dois anos, já descontada a taxa livre de risco (caderneta de poupança); $x_{1}$ e $x_{2}$ são os níveis de risco em um e dois anos; $x_{3}$ é o custo de administração; $u_{1}, u_{2}, v_{1}, v_{2}$ e $v_{3}$ são os parâmetros de ponderação específicos, atribuídos ao fundo $k$, que possibilitam a homogenização dos atributos desejáveis e dos atributos indesejáveis, maximizando a razão dada pela Equação [1]. A constante $c$ é uma característica do chamado retorno de escala variável (Banker, Charnes e Cooper, 1984) ${ }^{(1)}$. Para se obter os parâmetros de ponderação, é necessário resolver a formulação matemática que segue.

$$
\begin{aligned}
\text { Minimizar } & \sum_{i=1}^{3} v_{i k} x_{i k}+c \\
\text { Sujeito a : } & \sum_{r=1}^{2} u_{r k} y_{r k}=n \\
& -\sum_{r=1}^{2} u_{r k} y_{r j}+\sum_{i=1}^{3} v_{i k} x_{i j}+c \geq 0 \quad \text { para } j=1,2, \ldots, 106 .
\end{aligned}
$$

$\mathrm{Na}$ formulação [2] procuram-se os valores dos parâmetros de ponderação $v_{i}$ (onde $i=1,2,3$ ) que irão minimizar o denominador da Equação [1]. A primeira restrição identifica os parâmetros de ponderação $u_{r}$ (onde $r=1,2$ ) associados ao retorno médio em um ano e ao retorno médio em dois anos, numerador da Equação [1], estando esse limitado a um valor predeterminado $n$ (onde $n$ tem por objetivo fixar um limite ao numerador de [1] e pode assumir valores de 1, 10, 100 etc). A segunda restrição assegura que nenhum outro fundo de investimento, utilizando os parâmetros do fundo $k$, terá eficiência superior a $100 \% ; y_{r j}$ e $x_{i j}$ são os atributos desejáveis e atributos indesejáveis dos demais fundos (onde $j=1,2, \ldots, 106$ ). Obviamente, as ponderações $v_{i}$ e $u_{r}$ devem ser positivas. 
Observa-se que, quanto maior for a soma do retorno ponderado por unidade ponderada de risco e custo, melhor será o desempenho do fundo e que isso está de acordo com a teoria de finanças no relativo ao investidor racional, onde ele procura a máxima recompensa para determinado nível de risco, ou seja, procura maximizar a sua função utilidade esperada.

$\mathrm{Na}$ realização dos cálculos da eficiência para cada fundo de investimento, é possível obter-se, para algum parâmetro associado aos atributos desejáveis ou atributos indesejáveis, uma ponderação muito próxima a zero. Nesse caso, estarse-ia ignorando a existência desse atributo, o que poderia ser indesejável em alguns casos. Por exemplo, se um fundo de investimento possuir um custo de administração muito alto e receber ponderação muito próxima a zero, ele será ignorado, e isso poderá classificar o fundo como eficiente, mesmo apresentando um atributo totalmente indesejável.

Para evitar essa possibilidade de ocorrência, foram adicionadas restrições aos parâmetros calculados, de tal maneira que eles variem no intervalo de um a nove $(1 \leq$ parâmetros $\leq 9)$. Essas restrições são empíricas. Elas permitem que um atributo seja considerado no máximo nove vezes mais do que outro. Os limites mínimo e máximo dentro dos quais oscilarão os parâmetros de ponderação devem ser definidos especificamente pelo investidor, conforme o seu juízo de valor e a sua função utilidade esperada. Esse assunto é bem detalhado em Thompson et al. (1990) e Wong e Beasley (1990).

\section{Dados e Aspectos Metodológicos}

Os dados utilizados para o desenvolvimento deste trabalho foram obtidos na publicação Exame (1999) e no jornal Gazeta Mercantil (1997-1999). A amostra é constituída de 106 fundos mútuos de investimento carteira livre e abrange observações mensais no período de dezembro de 1997 a novembro de 1999. As informações referem-se ao retorno mensal médio em um ano (dezembro de 1998 novembro de 1999); retorno mensal médio em dois anos (dezembro de 1997 novembro de 1999); desvio padrão em um ano; desvio padrão em dois anos e o custo de administração (parcela fixa). A Tabela 1 apresenta a estatística descritiva dessas informações. Os dois primeiros itens são considerados como atributos desejáveis e os três últimos, atributos indesejáveis. A idéia básica é de que os investidores desejam os fundos que apresentem o maior nível de atributos desejáveis para um nível específico de atributos indesejáveis, ou seja, maximizem uma função utilidade esperada composta por cinco atributos. 


\section{Tabela 1: Estatística Descritiva das Taxas de Retorno Médio Mensal, Medida de Risco e Custo de Administração de 106 Fundos de Investimento Carteira Livre - Dezembro de 1997 a Novembro de 1999}

\begin{tabular}{|c|c|c|c|c|c|}
\hline $\begin{array}{l}\text { Estatística } \\
\text { Descritiva }\end{array}$ & $\begin{array}{c}\text { Retorno } \\
\text { médio } \\
1 \text { ano } \\
\left(\mathrm{y}_{1}\right)\end{array}$ & $\begin{array}{c}\text { Retorno } \\
\text { médio } \\
2 \text { anos } \\
\left(\mathrm{y}_{2}\right)\end{array}$ & $\begin{array}{c}\text { Risco } \\
1 \text { ano } \\
\left(\mathrm{x}_{1}\right)\end{array}$ & $\begin{array}{c}\text { Risco } \\
2 \text { anos } \\
\left(\mathrm{x}_{2}\right)\end{array}$ & $\begin{array}{c}\text { Custo } \\
\text { Adm. } \\
\left(\mathrm{x}_{3}\right)\end{array}$ \\
\hline Média Aritmética & 3,68 & 1,62 & 11,63 & 13,55 & 2,31 \\
\hline Mediana & 3,41 & 1,51 & 11,92 & 13,80 & 2,00 \\
\hline Valor Mínimo & 0,41 & 0,34 & 3,47 & 5,17 & 0,00 \\
\hline Valor Máximo & 8,62 & 3,73 & 19,00 & 20,58 & 8,00 \\
\hline Desvio Padrão & 1,31 & 0,74 & 2,23 & 2,12 & 1,55 \\
\hline
\end{tabular}

Obs: todos os valores estão em percentual (\%).

Os valores do retorno mensal médio, observados na Tabela 1, no período mais recente, por serem muito superiores aos do período todo, o que, somado ao risco que é inferior, tornam os últimos doze meses um período muito atrativo aos investidores. Esse fato ajuda a esclarecer a situação em que os resultados mais recentes, favoráveis ou desfavoráveis, acabam por exercer maior influência sobre a tomada de decisão. Outro fato importante é a grande oscilação do custo de administração. Ele varia de $0,00 \%$ até no máximo $8,00 \%$ ao ano; este último representa aproximadamente mais de quatro meses $(8,00 / 1,62=4,95)$ de remuneração mensal média dos fundos nos últimos dois anos.

Obviamente, não se está afirmando que são esses os atributos que os investidores utilizam para avaliar e selecionar um fundo de investimento. Muitas outras informações poderiam ser utilizadas, como, por exemplo, mais de uma medida de risco para cada fundo (desvio padrão e semivariância) ou, também, considerar a necessidade de investimento mínimo exigido pela maioria dos fundos. Enfim, são vários os atributos que podem ser utilizados. Cabe a cada investidor identificar os atributos de sua função utilidade esperada específica a ser maximizada.

Há grande possibilidade de ocorrer multicolinearidade entre as taxas de retorno médio, medido no período de um ano e dois anos, e entre o desvio padrão de um ano e dois anos, devido aos primeiros estarem contidos nos segundos. Em modelos estatísticos, é reconhecido que a multicolinearidade causa problemas, mas, na análise por envoltório de dados, não há maiores conseqüências, já que não há necessidade de testes de significância sobre os parâmetros obtidos. 


\section{Resultados Obtidos}

Por meio dos cálculos do índice ponderado de performance para cada um dos 106 fundos de investimento carteira livre, identificaram-se apenas sete fundos eficientes, os quais apresentam uma combinação de atributos desejáveis (taxas de retorno) e atributos indesejáveis (risco e custo) que dominam os demais, na perspectiva do investidor que considere tais atributos na sua função utilidade. A Tabela 2 apresenta, na primeira coluna, os sete fundos eficientes; na última coluna, os seus respectivos índices de eficiência e, nas colunas intermediárias, os seus atributos desejáveis e atributos indesejáveis.

\section{Tabela 2: Fundos de Investimento Eficientes - Dezembro de 1997 a Novembro de 1999}

\begin{tabular}{l|cccccc}
\hline \multicolumn{1}{c|}{ Fundos } & $\begin{array}{c}\text { Retorno } \\
\text { médio } \\
1 \text { ano } \\
\left(\mathrm{y}_{1}\right)\end{array}$ & $\begin{array}{c}\text { Retorno } \\
\text { médio } \\
2 \text { anos } \\
\left(\mathrm{y}_{2}\right)\end{array}$ & $\begin{array}{c}\text { Risco } \\
(\text { ano } \\
\left(\mathrm{x}_{1}\right)\end{array}$ & $\begin{array}{c}\text { Risco } \\
2 \text { anos } \\
\left(\mathrm{x}_{2}\right)\end{array}$ & $\begin{array}{c}\text { Custo } \\
\text { Adm. } \\
\left(\mathrm{x}_{3}\right)\end{array}$ & $\begin{array}{c}\text { Eficiência } \\
\text { IPP }\end{array}$ \\
\hline Safra Private & 5,42 & 3,64 & 12,67 & 15,41 & 1,00 & 100,00 \\
Dynamo Cougar & 7,83 & 3,12 & 8,37 & 11,00 & 2,00 & 100,00 \\
Pactual Fenix & 4,99 & 2,93 & 6,74 & 10,30 & 2,00 & 100,00 \\
Chase Small Cap & 3,51 & 0,34 & 6,73 & 8,98 & 0,75 & 100,00 \\
Aquarius FI - Santa Fé & 2,99 & 1,19 & 6,11 & 7,20 & 1,50 & 100,00 \\
Safra Multidividendos & 4,44 & 1,37 & 7,91 & 9,03 & 1,00 & 100,00 \\
Tatica Strategy CL & 1,79 & 1,33 & 3,47 & 5,17 & 3,00 & 100,00 \\
\hline
\end{tabular}

Obs: todos os valores estão em percentual (\%).

Observa-se na Tabela 2 que existe grande diversidade entre os atributos dos fundos de investimento eficientes. Por exemplo, o fundo Dynamo Cougar supera os demais fundos, no que se refere ao retorno em um ano (7,83\%), mas é superado pelo Safra Private no período de dois anos $(3,64 \%)$. Outro exemplo é o fundo Chase Small Cap. Esse fundo apresentou um excelente desempenho no último ano; mas, se for considerado apenas o resultado no período todo (2 anos), o seu desempenho passa a ser péssimo. No entanto, mediante o IPP, todos esses fundos têm o mesmo desempenho, devido ao fato de que, na maioria dos casos, os atributos de um fundo são ponderados diferentemente dos atributos de outros fundos, ou seja, um mesmo atributo (informação) tem diferentes graus de influência sobre a tomada de decisão de diferentes investidores. Na prática, essas 
diferenciações estão diretamente relacionadas ao conjunto de atributos utilizado pelos investidores, o que torna a seleção de um investimento uma decisão de caráter individual e muito específica.

Graficamente, os sete fundos eficientes estão situados acima dos demais, formando a fronteira eficiente. Como foram considerados dois atributos desejáveis e três atributos indesejáveis, a fronteira, na verdade, está situada num espaço de cinco dimensões (no plano podem ser representados no máximo dois atributos). Em termos didáticos, essa fronteira pode ser convertida para um plano, utilizando-se a soma ponderada dos atributos desejáveis e a soma ponderada dos atributos indesejáveis. Ela passa a ser uma linha que identifica a justa relação de troca entre atributos desejáveis e atributos indesejáveis, ou seja, uma linha de equilíbrio para os investidores. A Figura 1 apresenta graficamente essa relação de equilíbrio. No eixo das abscissas, são representados os atributos indesejáveis e, no eixo das ordenadas, os atributos desejáveis.

\section{Figura 1: Linha de Equilíbrio entre Atributos Desejáveis e Atributos Indesejáveis para os 106 Fundos de Investimento - Dezembro de 1997 a Novembro de 1999}

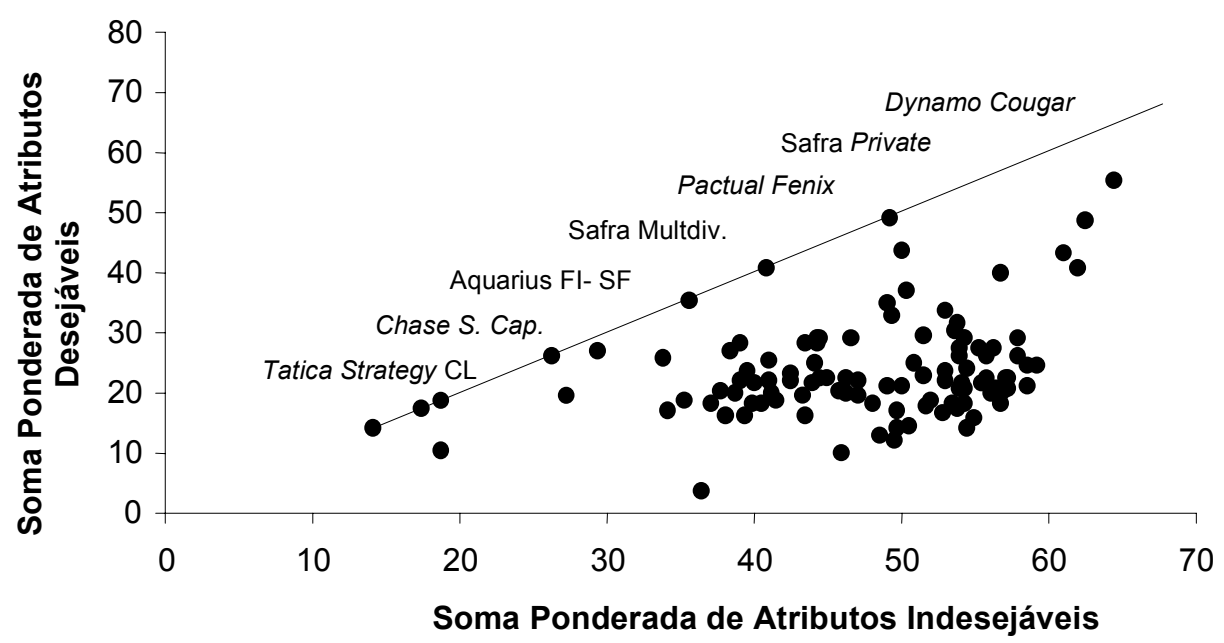

Como foi dito anteriormente, os fundos que estão sobre a linha de equilíbrio são eficientes (IPP $=100,00 \%$ ) e dominam os demais; os que estão abaixo dessa linha são considerados ineficientes ou dominados (IPP $<100,00 \%$ ). Os fundos que estão sobre a linha e próximos da origem dos eixos apresentam menores quantidades de atributos desejáveis e atributos indesejáveis, enquanto os mais 
afastados apresentam maiores quantidades de atributos desejáveis e atributos indesejáveis, sendo mantida, porém, uma relação justa de troca entre eles.

A Tabela 3 apresenta os sete fundos de investimento menos eficientes da amostra e seus respectivos atributos desejáveis e atributos indesejáveis. Na última coluna da tabela estão os índices de eficiência. Eles identificam a eficiência do fundo, comparando-o com o melhor desempenho observado ou com um ponto virtual que apresente o mesmo nível de atributos indesejáveis e esteja sobre a linha de equilíbrio. Tabela 3: Fundos de Investimento Menos Eficientes -
Dezembro de 1997 a Novembro de 1999

\begin{tabular}{l|cccccc}
\hline \multicolumn{1}{c|}{ Fundos } & $\begin{array}{c}\text { Retorno } \\
\text { médio } \\
1 \text { ano } \\
\left(\mathrm{y}_{1}\right)\end{array}$ & $\begin{array}{c}\text { Retorno } \\
\text { médio } \\
\left(\mathrm{y}_{2}\right)\end{array}$ & $\begin{array}{c}\text { Risco } \\
1 \text { ano } \\
\left(\mathrm{x}_{1}\right)\end{array}$ & $\begin{array}{c}\text { Risco } \\
2 \text { anos } \\
\left(\mathrm{x}_{2}\right)\end{array}$ & $\begin{array}{c}\text { Custo } \\
\text { Adm. } \\
\left(\mathrm{x}_{3}\right)\end{array}$ & $\begin{array}{c}\text { Eficiência } \\
\text { IPP }\end{array}$ \\
\hline BB - Ações Master & 2,72 & 0,79 & 12,13 & 13,78 & 4,00 & 28,85 \\
Novação Ações CL & 2,36 & 0,80 & 9,29 & 11,27 & 2,80 & 28,71 \\
Boavista CL & 2,35 & 0,51 & 10,90 & 12,80 & 2,00 & 26,45 \\
BB - Ações Eletrobrasil & 2,54 & 0,61 & 13,59 & 15,35 & 2,00 & 26,00 \\
BB - Ações Telebrasil & 2,23 & 0,42 & 11,80 & 14,09 & 2,00 & 24,00 \\
Safra Setorial Energia & 1,81 & 0,37 & 14,45 & 15,88 & 1,00 & 21,42 \\
Stock Asset & 0,41 & 0,47 & 11,48 & 13,00 & 2,00 & 10,78 \\
\hline
\end{tabular}

Obs: todos os valores estão em percentual (\%).

O inverso do IPP (IPP-1) identifica qual a magnitude da alteração que seria necessária nos atributos desejáveis, para que o fundo de investimento fosse eficiente, mantendo o seu atual nível de atributos indesejáveis. Por exemplo, o fundo Boavista CL apresenta IPP $=26,45 \%$. Para ele ser eficiente, deveria ter apresentado retorno médio no período de um ano de $8,88 \%\left(2,35 \times 0,2645^{-1}\right)$ e retorno médio no período de dois anos de $1,93 \%\left(0,51 \times 0,2645^{-1}\right)$.

A Tabela 4 apresenta a relação dos parâmetros de ponderação obtidos para o cálculo do índice ponderado de performance dos fundos eficientes e dos menos eficientes $^{(2)}$. Estas informações e aquelas apresentadas nas Tabelas 2 e 3 adicionadas da constante $c$, da formulação [2], formam a estrutura da Figura 1. 
Tabela 4: Parâmetros de Ponderação dos Atributos para os

Fundos de Investimento Eficientes e Menos Eficientes Dezembro de 1997 a Novembro de 1999

\begin{tabular}{l|ccccc}
\hline Fundos de investimento eficientes & & & & \\
\hline \multicolumn{1}{c}{ Fundos } & $\begin{array}{c}\text { Retorno } \\
\text { médio } \\
1 \text { ano } \\
\left(\mathrm{u}_{1}\right)\end{array}$ & $\begin{array}{c}\text { Retorno } \\
\text { médio } \\
2 \text { anos } \\
\left(\mathrm{u}_{2}\right)\end{array}$ & $\begin{array}{c}\text { Risco } \\
1 \text { ano } \\
\left(\mathrm{v}_{1}\right)\end{array}$ & $\begin{array}{c}\text { Risco } \\
2 \text { anos } \\
\left(\mathrm{v}_{2}\right)\end{array}$ & $\begin{array}{c}\text { Custo } \\
\text { Adm. } \\
\left(\mathrm{v}_{3}\right)\end{array}$ \\
\hline Safra Private & 1,48 & 9,00 & 1,33 & 1,00 & 8,95 \\
Dynamo Cougar & 2,71 & 9,00 & 1,00 & 1,00 & 1,00 \\
Pactual Fenix & 1,86 & 9,00 & 3,86 & 1,00 & 9,00 \\
Chase Small Cap & 4,79 & 1,54 & 1,00 & 1,00 & 9,00 \\
Aquarius FI - Santa Fé & 2,71 & 9,00 & 1,00 & 6,25 & 9,00 \\
Safra Multidividendos & 5,36 & 1,72 & 1,00 & 1,00 & 9,00 \\
Tatica Strategy CL & 7,10 & 1,00 & 1,00 & 6,99 & 1,00 \\
\hline Fundos de investimento menos eficientes & & & & 1,00 \\
\hline BB - Ações Master & 5,52 & 1,00 & 1,00 & 1,00 & 1,00 \\
Novação Ações CL & 5,69 & 1,00 & 1,00 & 1,00 & 1,00 \\
Boavista CL & 5,26 & 1,00 & 1,00 & 1,00 & 9,00 \\
BB - Ações Eletrobrasil & 5,34 & 1,00 & 1,00 & 1,00 & 1,00 \\
BB - Ações Telebrasil & 5,15 & 1,00 & 1,00 & 1,00 & 9,00 \\
Safra Setorial Energia & 5,22 & 1,00 & 1,00 & 1,00 & 9,00 \\
Stock Asset & 1,00 & 7,55 & 1,00 & 1,00 & 1,00 \\
\hline
\end{tabular}

A relação dos parâmetros de ponderação deixa claro que os fundos menos eficientes procuram enfocar mais o desempenho a curto prazo, no período de um ano. Esse fato é constatado pela ponderação superior atribuída ao retorno mensal médio em um ano. Já os fundos eficientes buscam uma melhor relação de dominância entre os resultados de curto e longo prazo, equilibrando o seu desempenho.

Por outro lado, uma coerente definição dos limites mínimo e máximo dos parâmetros de ponderação evita que fundos de investimento com valores extremos 
em um único atributo, apareçam como eficientes ou como um dos menos eficientes. Por exemplo, o fundo que apresentou o maior retorno médio no período de um ano $(8,62 \%)$ e o fundo que apresentou custo de administração igual a zero não foram classificados como eficientes, por apresentarem outros atributos indesejáveis, que foram devidamente ponderados. De forma idêntica, os fundos que apresentaram os maiores níveis de risco $(19,00 \%$ e $20,00 \%$ em cada período) não estão na lista dos menos eficientes, por apresentarem outros atributos desejáveis que, também, foram devidamente ponderados.

Uma comparação do índice ponderado de performance - DEA com uma das mais populares medidas de avaliação de fundos, o índice de Sharpe, é apresentada na Tabela 5. Na primeira coluna desta tabela estão listados os sete melhores fundos (parte superior da tabela) e os sete piores fundos (parte inferior da tabela); na segunda coluna estão os índices de Sharpe calculados para cada um dos fundos; na última coluna o IPP e nas colunas intermediárias, o conjunto de atributos considerados. A análise a seguir é realizada somente para os fundos de investimento que estão listados entre os sete melhores, segundo o índice de Sharpe, mas que não estão na lista dos sete eficientes, segundo o IPP. Essa análise também é realizada para os piores fundos.

O fundo FMIA CCF Banking ficou bem colocado na relação retorno/risco em dois anos $(0,2206)$, mas deixou a desejar no curto prazo; a relação retorno/risco em um ano ficou abaixo de seus concorrentes e isso prejudicou o seu desempenho pelo IPP. O Opportunity Logica II CL apresentou as maiores médias de retorno nos dois períodos considerados $(8,62 \%$ e $3,73 \%)$; porém o retorno do último ano não foi suficiente para compensar o elevado nível de risco a que submeteu os investidores (17,98\%). Outro fato que contribuiu para a sua exclusão entre os sete mais eficientes (através do IPP) foi o seu custo de administração de 4,00\%aa. O Santander Power apresentou boa remuneração média nos dois períodos; mas, nesse caso, o que muito prejudicou o desempenho do fundo foi o seu elevado custo de administração de 4,00\%aa.

Já para os piores fundos (segundo índice de Sharpe) Bandeirantes Setorial, Porto Seguro FMIA CL e Magliano, o que os exclui da lista dos sete menos eficientes (através do IPP), sem dúvida, foram os seus resultados no último ano, um pouco melhores do que aqueles dos seus concorrentes, permitindo, assim, melhorar o seu desempenho.

Embora existam diferenças, observa-se que, tanto para os melhores quanto para os piores fundos de investimento, existe uma sintonia entre o índice de Sharpe e o IPP; porém, como o IPP considera um conjunto mais amplo de informações, até mesmo englobando o próprio índice de Sharpe, tende a oferecer um resultado mais consistente ao investidor. 
Tabela 5: Os Melhores e os Piores Fundos de Investimentos segundo o Índice de Sharpe - Dezembro de 1997 a Novembro de 1999

\begin{tabular}{|c|c|c|c|c|c|c|c|}
\hline \multicolumn{8}{|l|}{ Melhores fundos } \\
\hline Fundos & $\begin{array}{l}\text { Índice de } \\
\text { Sharpe (a) }\end{array}$ & $\begin{array}{c}\text { Retorno } \\
\text { médio } \\
1 \text { ano } \\
\left(\mathrm{y}_{1}\right)\end{array}$ & $\begin{array}{c}\text { Retorno } \\
\text { médio } \\
2 \text { anos } \\
\left(\mathrm{y}_{2}\right) \\
\end{array}$ & $\begin{array}{c}\text { Risco } \\
1 \text { ano } \\
\left(\mathrm{x}_{1}\right)\end{array}$ & $\begin{array}{c}\text { Risco } \\
2 \text { anos } \\
\left(x_{2}\right)\end{array}$ & $\begin{array}{l}\text { Custo } \\
\text { Adm. } \\
\left(\mathrm{x}_{3}\right)\end{array}$ & $\begin{array}{c}\text { Eficiência } \\
\text { IPP }\end{array}$ \\
\hline Dynamo Cougar & 0,2682 & 7,83 & 3,12 & 8,37 & 11,00 & 2,00 & 100,00 \\
\hline Pactual Fenix & 0,2680 & 4,99 & 2,93 & 6,74 & 10,30 & 2,00 & 100,00 \\
\hline Safra Private & 0,2310 & 5,42 & 3,64 & 12,67 & 15,41 & 1,00 & 100,00 \\
\hline FMIA CCF Banking & 0,2206 & 3,12 & 2,93 & 10,40 & 12,92 & 1,00 & 92,80 \\
\hline Opportunity L. II CL & 0,2144 & 8,62 & 3,73 & 17,98 & 15,86 & 4,00 & 86,20 \\
\hline Tatica Strategy CL & 0,2089 & 1,79 & 1,33 & 3,47 & 5,17 & 3,00 & 100,00 \\
\hline Santander Power & 0,2003 & 5,01 & 3,27 & 12,80 & 14,68 & 4,00 & 74,00 \\
\hline \multicolumn{8}{|l|}{ Piores fundos } \\
\hline Boavista CL & 0,0266 & 2,35 & 0,51 & 10,90 & 12,80 & 2,00 & 26,45 \\
\hline Bandeirantes Set. & 0,0244 & 2,61 & 0,64 & 10,48 & 12,68 & 4,00 & 29,00 \\
\hline Stock Asset & 0,0231 & 0,41 & 0,47 & 11,48 & 13,00 & 2,00 & 10,78 \\
\hline Safra Set. Energia & 0,0183 & 1,81 & 0,37 & 14,45 & 15,88 & 1,00 & 21,42 \\
\hline BB Ações Telebrasil & 0,0177 & 2,23 & 0,42 & 11,80 & 14,09 & 2,00 & 24,00 \\
\hline Porto Seguro CL & 0,0122 & 3,70 & 0,41 & 11,90 & 13,13 & 3,00 & 38,52 \\
\hline Magliano & 0,0015 & 3,41 & 0,35 & 12,46 & 13,48 & 4,00 & 34,06 \\
\hline
\end{tabular}

(a) Para o cálculo do índice de Sharpe foi subtraída, do retorno mensal médio em dois anos, uma parcela equivalente a 1/12 do custo de administração; após, dividiu-se esse resultado pelo risco em 2 anos.

\section{Conclusão}

O objetivo deste estudo foi apresentar uma nova aplicação da técnica de análise por envoltório de dados. Ela foi usada para investigar e analisar comparativamente o desempenho de 106 fundos de investimento carteira livre, calculando-se um índice ponderado de performance. Essa metodologia exclui a necessidade de uma definição prévia do padrão de comparação e da utilização de um índice representativo do mercado. $\mathrm{O}$ índice ponderado de performance se mostra adequado à seleção de investimentos, quando os atributos utilizados forem além dos dois fatores tradicionais, risco e retorno, utilizados pela teoria de finanças.

Para a amostra considerada com a função utilidade definida, sete fundos foram identificados como eficientes. Esses fundos apresentam uma justa relação de 
troca entre atributos desejáveis e atributos indesejáveis, além de possuírem um conjunto de atributos que não pode ser superado por nenhum outro fundo da amostra. Comparando-se os parâmetros de ponderação dos fundos eficientes com os dos fundos menos eficientes, conclui-se que os menos eficientes procuram enfocar uma relação de dominância nos resultados de curto prazo. Outra constatação é que atributos de valores extremos não definem o melhor ou pior desempenho por estarem associados a outros atributos e ponderações indesejáveis ou desejáveis.

A análise por envoltório de dados permite ao investidor não somente tomar decisões sobre em quais fundos investir, mas também mostra as razões de não se investir em outros fundos. Antes de tudo, a análise por envoltório de dados é ferramenta gerencial que se deixa moldar segundo os critérios específicos da função utilidade esperada de cada investidor, auxiliando, assim, o processo de tomada de decisão ${ }^{(3)}$.

\section{Notas}

${ }^{1}$ O retorno de escala variável tem como principal finalidade tornar a avaliação mais flexível, pois não exige que seja mantida uma relação de proporcionalidade entre atributos desejáveis e atributos indesejáveis nos diversos níveis de operação.

${ }^{2}$ Também foi calculado o IPP sem as restrições adicionais aos parâmetros de ponderação. Nesse caso, foram identificados onze fundos de investimento eficientes. Para alguns fundos, a relação de superioridade da ponderação de um atributo sobre os demais foi de até 125 vezes, o que justifica a definição de limites mínimo e máximo.

${ }^{3} \mathrm{O}$ presente trabalho foi elaborado enquanto o segundo autor realizava estágio de pós-doutoramento na Columbia Business School, USA, sob o patrocínio da Fundação CAPES e da Comissão Fulbright.

\section{ReferÉnCiAs Bibliográficas}

BANKER, R. D.;

CHARNES, A.;

COOPER, W. W.

Some models for estimating technical and scale inefficiencies in data envelopment analysis. Management Science, v. 30, $\mathrm{n}$. 9, p. 1078-1092, 1984. 
CHARNES, A.;

COOPER, W. W.;

RHODES, E.

Measuring efficiency of decision making units. European Journal of Operational Research, v. 2, n. 6, p. 429-444, 1978.

EXAME.

Guia dos melhores fundos de investimento. São Paulo : [s.n.], 1999.

FISHER, K. L.;

STATMAN, R.

The mean-variance-optimization puzzle: security portfolios and food portfolios. Financial Analysts Journal, v. 53, n. 4, p. 41-50, 1997.

GAZETA MERCANTIL.

São Paulo : [s.n.], 1997-1999.

GRAHAN, J.;

HARVEY, C. R.

Market timing and volatility implied in investment newsletter's asset allocation recomendation. NBER - Working Paper, 1994.

GRINBLATT, M.;

TITMAN, S.

Performance measurement without benchmarks : an examination of mutual fund returns. Journal of Business, v. 66, p. 47-68, 1993.
JENSEN, M. C.

The performance of mutual funds in the period 1945-1964. Journal of Finance, v. 23, n. 2, p. 389-416, 1968.

MARKOWITZ, H. M.

Portfolio selection. Journal of

Finance, v. 7, n. 1, p. 77-91, 1952.

MCMULLEN, P. R.;

STRONG, R. A.

Selection of mutual funds using data envelopment analysis. Journal of Business and Economic Studies, v. 4, n. 1, p. 1-12, 1998.

SHARPE, W. F.

Mutual fund performance. Journal of Business, v. 39, n. 1, p. 119-138, 1966.

STRONG, R. A.

Portfolio construction, management and protection.

New York: West Publishers, 1993.

THOMPSON, R. G. et al.

The role of multiplier bounds in efficiency analysis with application to Kansas farming. Journal of Econometrics, v. 46, p. 93-108, 1990.

TREYNOR, J. L.

How to rate management of investment funds. Harvard Business Review, v. 43, n. 1, p. $63-75,1965$. 
WONG, Y. B.;

BEASLEY, J. E.

Restricting weight flexibility in data envelopment analysis. Journal of the Operational Research Society, v. 41, n. 9, p. 829-835, 1990. 